\title{
Fragmentation of charmed quark to double-charmed hadrons
}

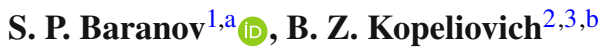 \\ ${ }^{1}$ P.N. Lebedev Institute of Physics, Lenin Avenue 53, 119991 Moscow, Russia \\ ${ }^{2}$ Departamento de Física, Universidad Técnica Federico Santa María, Valparaiso, Chile \\ ${ }^{3}$ Centro Científico-Tecnológico de Valparaíso, Avenida España 1680, Valparaiso, Chile
}

Received: 29 January 2021 / Accepted: 21 April 2021 / Published online: 1 May 2021

(C) The Author(s) 2021

\begin{abstract}
We consider the production of hadrons containing two charmed quarks in $p p$ and $e e$ collisions. We perform a numerical comparison of the fragmentation approach with the full calculation at $\mathcal{O}\left(\alpha_{s}^{4}\right)$. We conclude that the nonfragmentation contributions remain important up to transverse momenta as large as about $40 \mathrm{GeV}$, thus making questionable the applicability of the fragmentation approximation at smaller transverse momenta.
\end{abstract}

\section{Introduction}

The factorization principle and the concept of quark and gluon fragmentation functions [1] constitute a widely exploited framework to describe particle production phenomena at collider energies (e.g., see [2-5]). Over the years, large efforts have been invested in the theoretical calculation and experimental determination of the various fragmentation functions. In some important cases, such as the production of heavy quarkonium states, the relevant fragmentation functions are believed to be calculable with QED and QCD perturbative methods. For example, the heavy quark fragmentation to a heavy pair $Q \rightarrow Q \bar{Q}$ is known at the Next-to-Leading Power (NLP) accuracy [6].

Despite the method is proved to be mathematically consistent for asymptotically high transverse momenta of the produced particles, the real conditions may not meet this asymptotic regime. So, it is certainly of great interest to outline the kinematic domain where the fragmentation approach can be trusted as a reliable approximation. This issue has been previously studied in a number of papers. Reference [7] addresses the production of quarkonium states in $e^{+} e^{-}$annihilation; Ref. [8] focuses on the gluon fragmentation in $p p$ collisions; Ref. [9] considers quarks fragmenting into colorsinglet $Q \bar{Q}$ states and gluons fragmenting into color-octet

\footnotetext{
a e-mail: baranovsp@ lebedev.ru (corresponding author)

be-mail: boris.kopeliovich@usm.cl
}

states. The moral deduced from the above studies is that the energy at which the fragmentation result becomes reliable may exceed the quarkonium mass by more than one order of magnitude. However, the value of the required energy is not universal, and the validity of fragmentation predictions "must be carefully checked on a case-by-case basis" [7].

Our previous study [10] was devoted to the production of $J / \psi$ mesons in proton-proton collisions, and the conclusions were consistent with Ref. [9]. Now we extend the consideration to hadrons with other quantum numbers and to other colliding beams. Namely, we address the production of $\eta_{c}$ mesons and doubly-charmed $\Xi_{c c}$ baryons in protonproton collisions and, also, the production of $J / \psi$ mesons in lepton-lepton collisions via two-photon subprocess.

To carry out this task, we make a comparison of two calculations. First, we consider an $\mathcal{O}\left(\alpha_{s}^{2}\right)$ subprocess $g g \rightarrow c \bar{c}$ (or $\gamma \gamma \rightarrow c \bar{c}$ ) and convolute it with an $\mathcal{O}\left(\alpha_{s}^{2}\right)$ fragmentation function $c \rightarrow \psi c$, where $\psi$ may stand for $\eta_{c}, \Xi_{c c}$, or $J / \psi$. Second, we perform a full $\mathcal{O}\left(\alpha_{s}^{4}\right)$ calculation for the process $g g \rightarrow \psi c \bar{c}$ and see to what extent does the 'full result' matches the fragmentation interpretation.

To avoid any confusion about the goal of the paper, we have to remind that our calculation of $\eta_{c}, \Xi_{c c}$, and $J / \psi$ associated production with $c+\bar{c}$ is not at all unique; and that the relevant fragmentation functions have even been calculated at the NLO accuracy (e.g., [11] for $c \rightarrow J / \psi$ and [12] for $c \rightarrow \Xi_{c c}$ ). But the point of our interest is not in these processes on their own. We see our practical result in establishing the applicability limits for the fragmentation approach. These limits have never been shown in the literature for the mentioned processes.

\section{Perturbative color-singlet fragmentation $c \rightarrow \psi c$}

To calculate the charmed quark fragmentation function, we start with the process $e^{+} e^{-} \rightarrow \gamma^{*} \rightarrow \psi c \bar{c}$ considered in the 

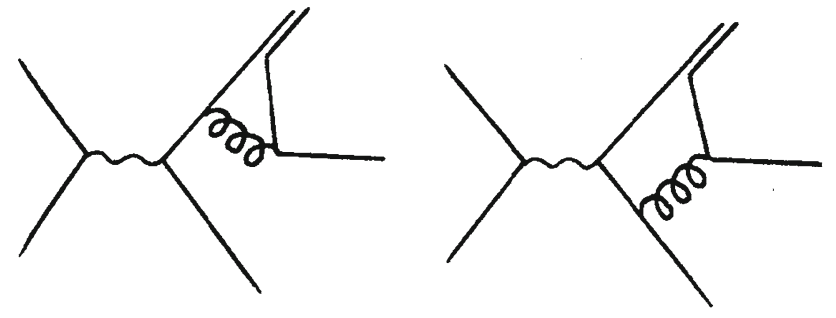

Fig. 1 Feynman diagrams used to calculate the $c \rightarrow \psi$ fragmentation function from $e^{+} e^{-}$annihilation, $e^{+} e^{-} \rightarrow \gamma^{*} \rightarrow \psi+c+\bar{c}$
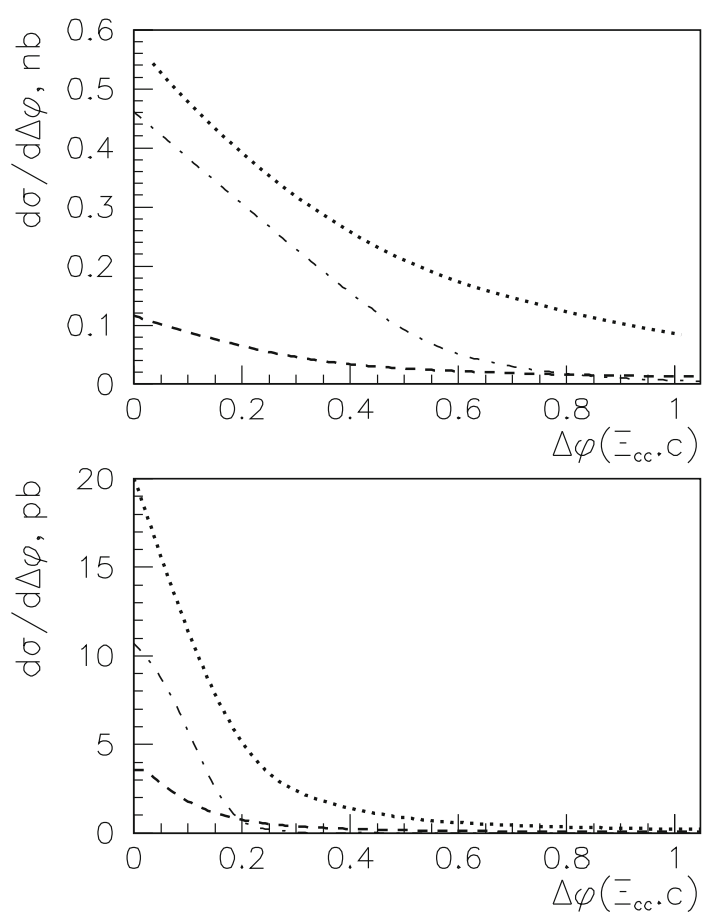

Fig. 2 The process $g+g \rightarrow \Xi_{c c}+\bar{c}+\bar{c}$; azimuthal angle difference between the $\Xi_{c c}$ baryon and the comoving $\bar{c}$-quark as seen under the different kinematic constraints. Upper panel: dotted, $p_{\Xi T}>5 \mathrm{GeV}$, $p_{T}^{*}>20 \mathrm{GeV}$; dashed, $p_{\Xi T}>20 \mathrm{GeV}, p_{T}^{*}>20 \mathrm{GeV}$; dash-dotted, $m^{*}<E^{*} / 3$. Lower panel: dotted, $p_{\Xi T}>20 \mathrm{GeV}, p_{T}^{*}>50 \mathrm{GeV}$; dashed, $p_{\Xi T}>50 \mathrm{GeV}, p_{T}^{*}>50 \mathrm{GeV}$; dash-dotted, $m^{*}<E^{*} / 10$

virtual photon rest frame with the $z$ axis oriented along the negative direction of the charmed antiquark momentum. The corresponding Feynman diagrams are displayed in Fig. 1.

The bound state quantum numbers are determined by the properly chosen projection operators in the production amplitudes. The production of $\Xi_{c c}$ baryons is dominated by the production of double-charm diquark $c c$ in the color antitriplet state. The diquark can then pick up a lighter quark from the vacuum and convert into a real baryon. The 4-momentum of the baryon can be approximately taken equal to the momentum of the heavy diquark. We have to consider the diquarks with spin $0 c c_{0}$ and $1 c c_{1}$.
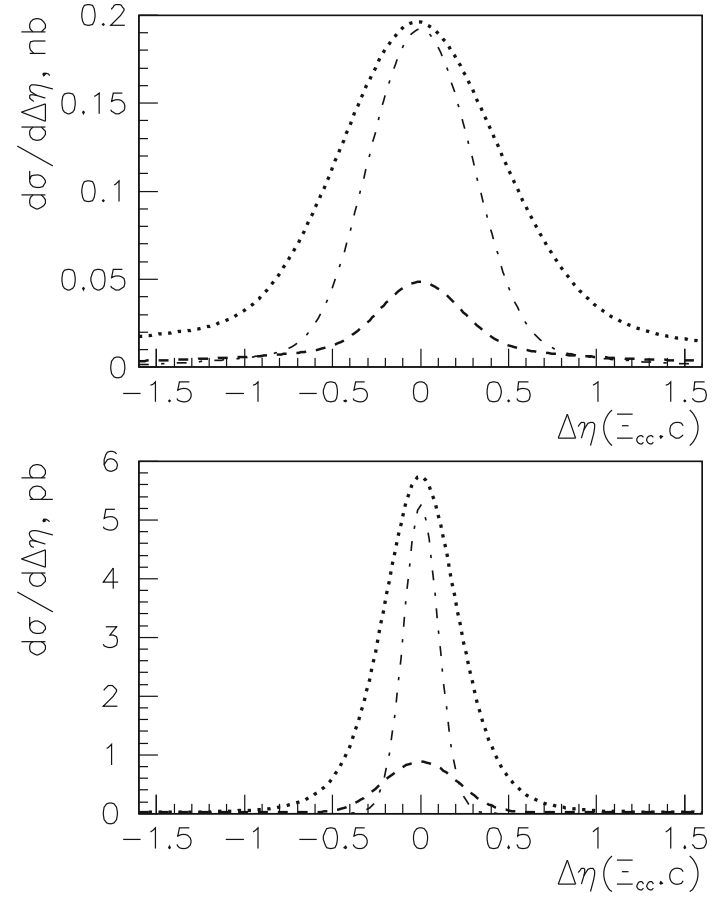

Fig. 3 The process $g+g \rightarrow \Xi_{c c}+\bar{c}+\bar{c}$; pseudorapidity difference between the $\Xi_{c c}$ baryon and the comoving $\bar{c}$-quark as seen under the different kinematic constraints. Upper panel: dotted, $p_{\Xi T}>5 \mathrm{GeV}$, $p_{T}^{*}>20 \mathrm{GeV}$; dashed, $p_{\Xi T}>20 \mathrm{GeV}, p_{T}^{*}>20 \mathrm{GeV}$; dash-dotted, $m^{*}<E^{*} / 3$. Lower panel: dotted, $p_{\Xi T}>20 \mathrm{GeV}, p_{T}^{*}>50 \mathrm{GeV}$; dashed, $p_{\Xi T}>50 \mathrm{GeV}, p_{T}^{*}>50 \mathrm{GeV}$; dash-dotted, $m^{*}<E^{*} / 10$

The fully differential cross section then reads

$$
\begin{aligned}
d \sigma= & \frac{1}{2 s} \frac{1}{(2 \pi)^{5}}\left|\mathcal{M}\left(e e \rightarrow \gamma^{*}\right)\right|^{2} \cdot\left|\mathcal{M}\left(\gamma^{*} \rightarrow \psi c \bar{c}\right)\right|^{2} \\
& \times \frac{1}{s^{2}} \frac{\lambda^{1 / 2}\left(s, p^{* 2}, m_{c}^{2}\right)}{8 s} \frac{\lambda^{1 / 2}\left(p^{* 2}, m_{\psi}^{2}, m_{c}^{2}\right)}{8 p^{* 2}} \\
& \times d \Omega d p^{* 2} d \phi d \cos \theta,
\end{aligned}
$$

where $s$ is the overall invariant energy; $p_{\psi}, p_{1}$ and $p_{2}$ the 4-momenta of final hadron and the charmed quark and antiquark, respectively; $\Omega, \phi$, and $\theta$ the angular variables of the reaction; $\lambda$ is the standard 'triangle' kinematic function [13]; and the momentum $p^{*}=p_{1}+p_{\psi}$ represents the fragmenting (or 'parent') quark momentum.

The above formula can be interpreted as a product of the quark production cross section

$$
\begin{aligned}
& d \sigma\left(e^{+} e^{-} \rightarrow c \bar{c}\right) \\
& \quad=\frac{1}{2 s} \frac{1}{(2 \pi)^{2}} \frac{\lambda^{1 / 2}\left(s, p^{* 2}, m_{c}^{2}\right)}{8 s}|\mathcal{M}(e e \rightarrow c \bar{c})|^{2} d \Omega
\end{aligned}
$$

and the $c$-quark fragmentation probability. After dividing Eq. (1) by Eq. (2) we arrive at the definition of the differential fragmentation function 

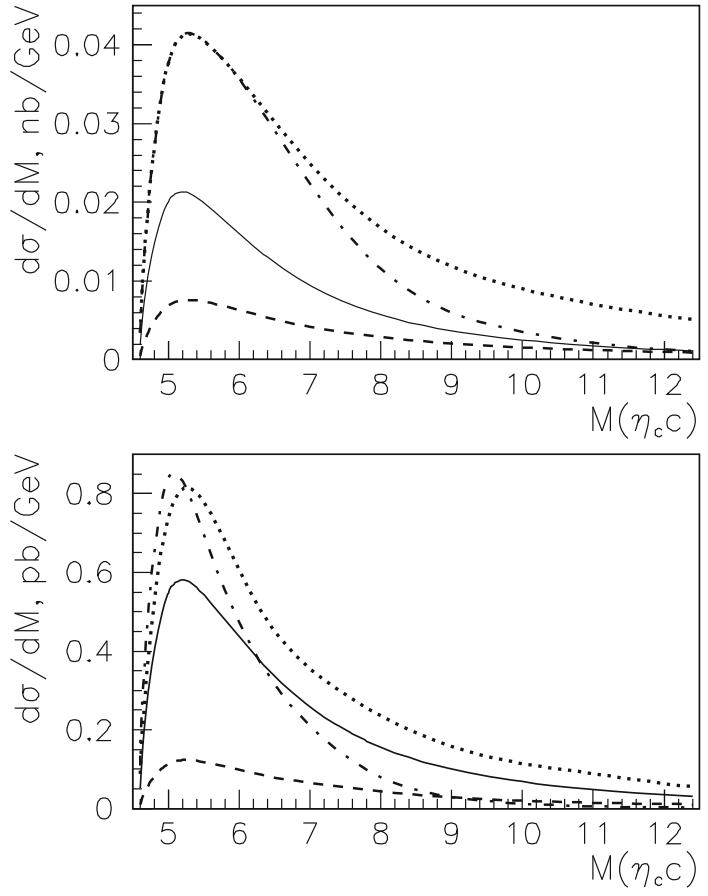

Fig. 4 The process $g+g \rightarrow \eta_{c}+c+\bar{c}$; invariant mass of the $\eta_{c}+c$ system as seen under the different kinematic constraints. Upper panel: dotted, $p_{\eta T}>5 \mathrm{GeV}, p_{T}^{*}>20 \mathrm{GeV}$; dashed, $p_{\eta T}>20 \mathrm{GeV}, p_{T}^{*}>$ $20 \mathrm{GeV}$; dash-dotted, $m^{*}<E^{*} / 3$; solid, factorization calculation for $p_{T}^{*}>20 \mathrm{GeV}$. Lower panel: dotted, $p_{\eta T}>20 \mathrm{GeV}, p_{T}^{*}>50 \mathrm{GeV}$; dashed, $p_{\eta T}>50 \mathrm{GeV}, p_{T}^{*}>50 \mathrm{GeV}$; dash-dotted, $m^{*}<E^{*} / 10$; solid, factorization calculation for $p_{T}^{*}>50 \mathrm{GeV}$

$$
\begin{aligned}
& d D\left(c^{*} \rightarrow \psi c\right) \\
& =\frac{1}{(2 \pi)^{3}} \frac{\left|\mathcal{M}\left(\gamma^{*} \rightarrow \psi c \bar{c}\right)\right|^{2}}{\left|\mathcal{M}\left(\gamma^{*} \rightarrow c \bar{c}\right)\right|^{2}} \lambda^{1 / 2}\left(p^{* 2}, m_{\psi}^{2}, m_{c}^{2}\right) d p^{* 2} d \phi d \cos \theta
\end{aligned}
$$

The latter can be further reduced to the conventional fragmentation function $D_{c / \psi}(z)$ by introducing the light-cone variable $z=p_{\psi}^{+} / p^{*+}=\left(E_{\psi}+p_{\psi, z}\right) /\left(E^{*}+p_{z}^{*}\right)$ and integrating over all other variables in Eq.(3):

$$
D_{c / \psi}(z)=\int D\left(c^{*} \rightarrow \psi c\right) \delta\left(z-p_{\psi}^{+} / p^{*+}\right) d p^{* 2} d \phi d \cos \theta .
$$

The full factorization takes place in the high energy limit, $\sqrt{s} \gg m_{c}$, when the terms of the order $m_{c} / \sqrt{s}$ become small and can be neglected. At finite energies the factorization is only approximate. It is the matter of our numerical study, to understand the kinematic conditions which make the factorization approximation applicable to the gluon-gluon fusion case.
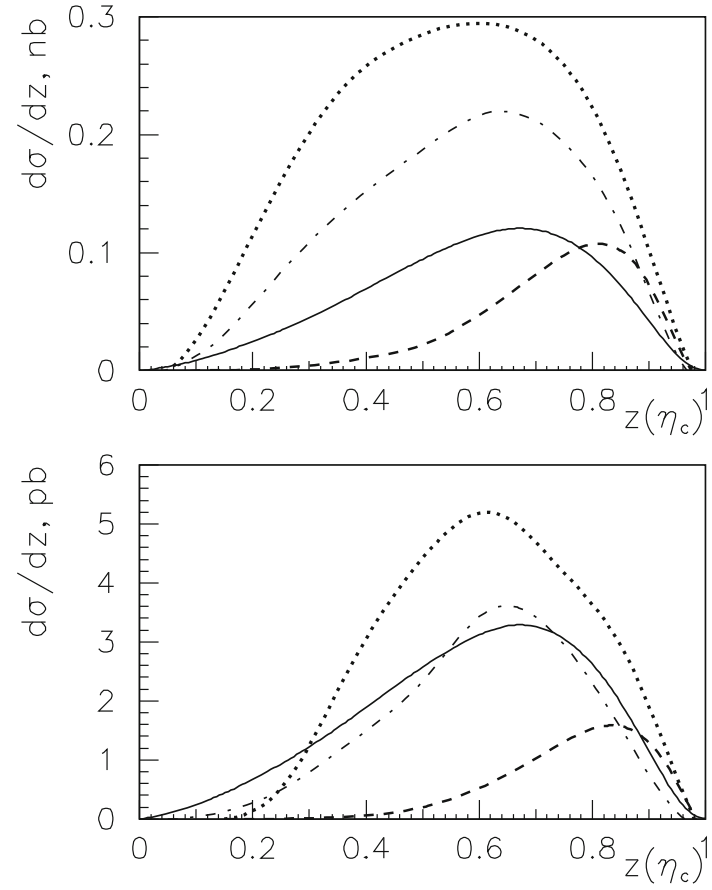

Fig. 5 The process $g+g \rightarrow \eta_{c}+c+\bar{c}$; the distributions over the fragmentation variable $z$ as seen under the different kinematic constraints. Upper panel: dotted, $p_{\eta T}>5 \mathrm{GeV}, p_{T}^{*}>20 \mathrm{GeV}$; dashed, $p_{\eta T}>$ $20 \mathrm{GeV}, p_{T}^{*}>20 \mathrm{GeV}$; dash-dotted, $m^{*}<E^{*} / 3$; solid, factorization calculation for $p_{T}^{*}>20 \mathrm{GeV}$. Lower panel: dotted, $p_{\eta T}>20 \mathrm{GeV}$, $p_{T}^{*}>50 \mathrm{GeV}$; dashed, $p_{\eta T}>50 \mathrm{GeV}, p_{T}^{*}>50 \mathrm{GeV}$; dash-dotted, $m^{*}<E^{*} / 10$; solid, factorization calculation for $p_{T}^{*}>50 \mathrm{GeV}$

\section{Full $\mathcal{O}\left(\alpha_{s}^{4}\right)$ calculation}

\subsection{Gluon-gluon fusion processes}

The calculation of the gluon-gluon fusion processes

$$
\begin{aligned}
& g+g \rightarrow \eta_{c}+c+\bar{c}, \\
& g+g \rightarrow \Xi_{c c}+\bar{c}+\bar{c}, \\
& \gamma+\gamma \rightarrow J / \psi+c+\bar{c}
\end{aligned}
$$

is based on, respectively, 42, 36, and 20 Feynman diagrams shown in Fig. 1 in Ref. [10]. They are all necessary to compose gauge invariant sets (for more details see [14,15], where one can find explicit algebraic expressions for all of these diagrams). The amplitudes for the production of vector $(J / \psi$ or $\left.c c_{1}\right)$ and (pseudo)scalar $\left(\eta_{c}\right.$ or $\left.c c_{0}\right)$ states contain different spin projection operators. The amplitudes for color singlet $\left(\mathrm{J} / \psi\right.$ or $\left.\eta_{c}\right)$ and color antitriplet (diquark) states employ different combinations of color coefficients. The evaluation of Feynman diagrams is straightforward and was done with the algebraic manipulation system FORM [16].

Having the heavy quarks produced, the probability to form a bound state is determined by a single parameter, the radial wave function at the origin $|\mathcal{R}(0)|^{2}$. It can be calculated within potential models or extracted from the particle decay 

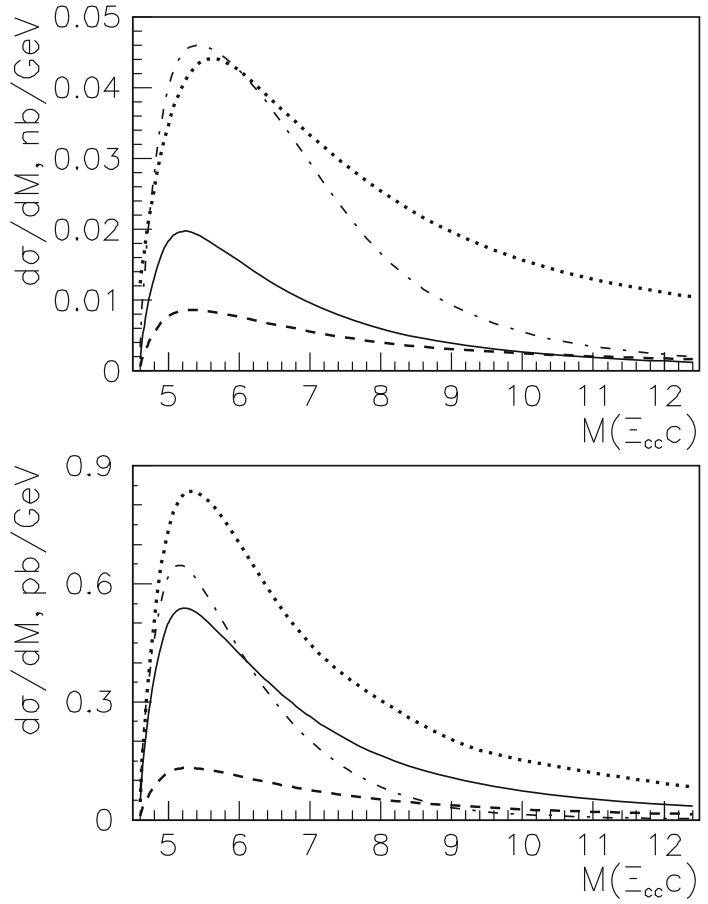

Fig. 6 The process $g+g \rightarrow \Xi_{c c}+\bar{c}+\bar{c}$; invariant mass of the $\Xi_{c c}+\bar{c}$ system as seen under the different kinematic constraints. Upper panel: dotted, $p_{\Xi T}>5 \mathrm{GeV}, p_{T}^{*}>20 \mathrm{GeV}$; dashed, $p_{\Xi T}>20 \mathrm{GeV}$, $p_{T}^{*}>20 \mathrm{GeV}$; dash-dotted, $m^{*}<E^{*} / 3$; solid, factorization calculation for $p_{T}^{*}>20 \mathrm{GeV}$. Lower panel: dotted, $p_{\Xi T}>20 \mathrm{GeV}, p_{T}^{*}>50 \mathrm{GeV}$; dashed, $p_{\Xi T}>50 \mathrm{GeV}, p_{T}^{*}>50 \mathrm{GeV}$; dash-dotted, $m^{*}<E^{*} / 10$; solid, factorization calculation for $p_{T}^{*}>50 \mathrm{GeV}$

widths. To be definite, we set $\left|\mathcal{R}_{\psi}(0)\right|^{2}=\left|\mathcal{R}_{\eta}(0)\right|^{2}=0.8$ $\mathrm{GeV}^{3},\left|\mathcal{R}_{\Xi}(0)\right|^{2}=0.4 \mathrm{GeV}^{3}$, although these values are irrelevant for our purposes. We only want to examine the agreement or disagreement between the 'full' and factorized calculations.

Summarizing, the fully differential cross section reads

$$
\begin{aligned}
& d \sigma(p p \rightarrow \psi c \bar{c} X) \\
& =\frac{\pi \alpha_{s}^{4}}{3 \hat{s}^{2}} \frac{|\mathcal{R}(0)|^{2}}{4 \pi} \frac{1}{4} \sum_{\text {spins }} \frac{1}{64} \sum_{\text {colors }}|\mathcal{M}(g g \rightarrow \psi c \bar{c})|^{2} \\
& \quad \times \mathcal{F}_{g}\left(x_{1}, \mu^{2}\right) \mathcal{F}_{g}\left(x_{2}, \mu^{2}\right) \\
& \quad \times d p_{\psi T}^{2} d p_{c T}^{2} d y_{\psi} d y_{c} d y_{\bar{c}} \frac{d \phi_{\psi}}{2 \pi} \frac{d \phi_{c}}{2 \pi},
\end{aligned}
$$

where $s$ is the total initial invariant energy squared, $\hat{s}$ the squared energy of the partonic subprocess, $x_{1}$ and $x_{2}$ the parton light-cone momentum fractions; $\mathcal{F}_{g}\left(x, \mu^{2}\right)$ the gluon distribution function in the proton; $\mu^{2}=\hat{s} / 4$; and $y_{\psi}, y_{c}$, $y_{\bar{c}}, p_{\psi T}, p_{c T}, p_{\bar{c} T}, \phi_{\psi}, \phi_{c}$ and $\phi_{\bar{c}}$ the rapidities, transverse momenta and azimuthal angles of the final particle $\psi$ and the accompanying charmed quark and antiquark, respectively.

We use the MSTW leading-order set [17] for the gluon densities in (5), (6) and Weizsäcker-Williams approximation $[18,19]$ for equivalent photon flux in (7). The multidimen-
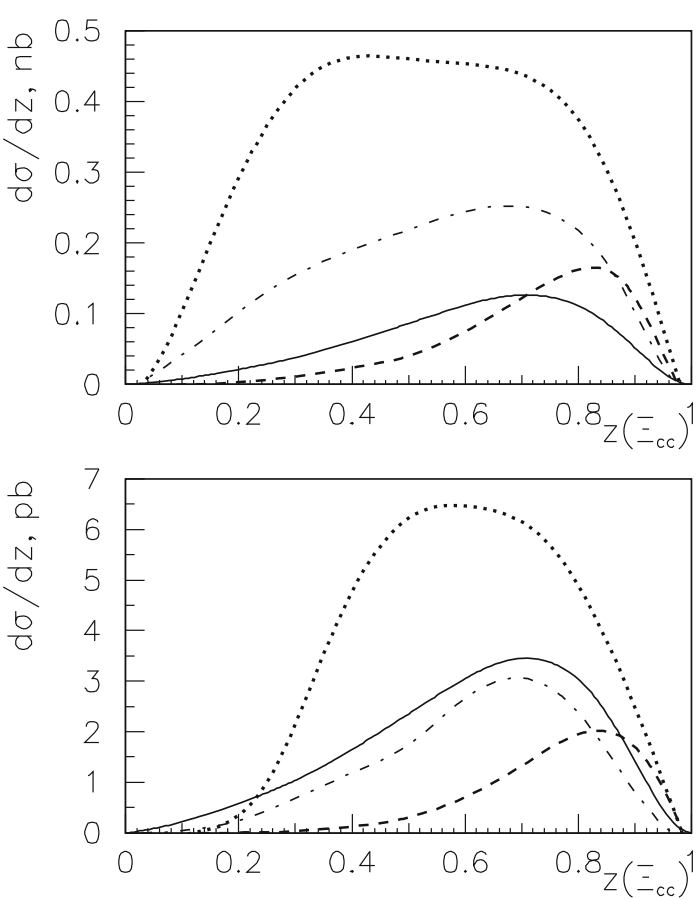

Fig. 7 The process $g+g \rightarrow \Xi_{c c}+\bar{c}+\bar{c}$; the distributions over the fragmentation variable $z$ as seen under the different kinematic constraints. Upper panel: dotted, $p_{\Xi T}>5 \mathrm{GeV}, p_{T}^{*}>20 \mathrm{GeV}$; dashed, $p_{\Xi T}>20 \mathrm{GeV}, p_{T}^{*}>20 \mathrm{GeV}$; dash-dotted, $m^{*}<E^{*} / 3$; solid, factorization calculation for $p_{T}^{*}>20 \mathrm{GeV}$. Lower panel: dotted, $p_{\Xi T}>20 \mathrm{GeV}, p_{T}^{*}>50 \mathrm{GeV}$; dashed, $p_{\Xi T}>50 \mathrm{GeV}$, $p_{T}^{*}>50 \mathrm{GeV}$; dash-dotted, $m^{*}<E^{*} / 10$; solid, factorization calculation for $p_{T}^{*}>50 \mathrm{GeV}$

sional integration in (8) has been performed by means of the Monte-Carlo technique, using the routine VEGAS [20].

\subsection{Theoretical experiment: "jet" reconstruction}

To reinterpret the results of 'full calculation' in terms of fragmentation approach we have to reconstruct the fragmenting quark momentum. In what follows we will refer to the $J / \psi+c+\bar{c}$ channel taking it as an example, but understand that the same applies to all other channels too. Accordingly, $p_{\psi}$ may denote the momentum of $J / \psi$ meson, or $\eta_{c}$ meson, or $\Xi_{c c}$ baryon.

We can associate the final state $J / \psi$ meson with either $c$ or $\bar{c}$, thus referring to the quark or antiquark fragmentation cases. We choose between these two possibilities by taking the configuration with the lowest two-body invariant mass: either $M(\psi c)<M(\psi \bar{c})$, or vice versa. Here we follow the same way as in [10].

Let the chosen system be the $\psi c$ (quark fragmentation). Then the momentum $p^{*}$ of the fragmenting quark is evidently $p^{*}=p_{\psi}+p_{c}$. The factorization hypothesis (or theorem) requires that the fragmenting quark transverse momentum be large enough, $p_{T}^{*}>p_{T, \min }$. In our numerical studies we tried 

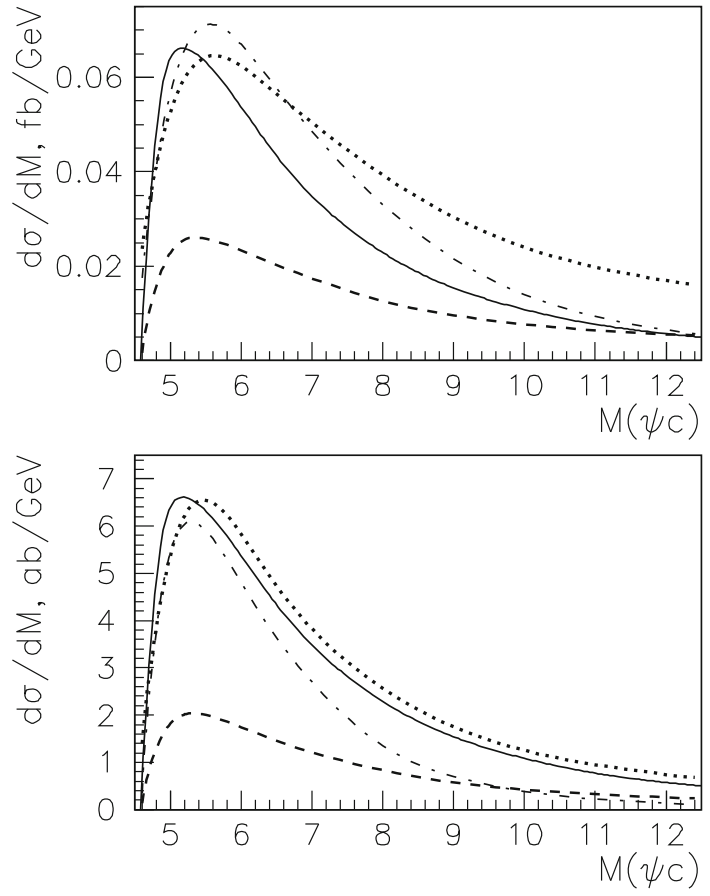

Fig. 8 The process $\gamma+\gamma \rightarrow J / \psi+c+\bar{c}$; invariant mass of the $J / \psi+c$ system as seen under the different kinematic constraints. Upper panel: dotted, $p_{\psi T}>5 \mathrm{GeV}, p_{T}^{*}>20 \mathrm{GeV}$; dashed, $p_{\psi T}>20 \mathrm{GeV}$, $p_{T}^{*}>20 \mathrm{GeV}$; dash-dotted, $m^{*}<E^{*} / 3$; solid, factorization calculation for $p_{T}^{*}>20 \mathrm{GeV}$. Lower panel: dotted, $p_{\psi T}>20 \mathrm{GeV}, p_{T}^{*}>50 \mathrm{GeV}$; dashed, $p_{\psi T}>50 \mathrm{GeV}, p_{T}^{*}>50 \mathrm{GeV}$; dash-dotted, $m^{*}<E^{*} / 10$. solid, factorization calculation for $p_{T}^{*}>50 \mathrm{GeV}$

$p_{T, \text { min }}=20 \mathrm{Gev}$ and $p_{T, \min }=50 \mathrm{GeV}$. Another necessary condition for the validity of fragmentation approach is that the invariant mass $m^{*}$ of the fragmenting system must be small in comparison with its energy $E^{*}$. In our numerical exercises we tried $m^{*}<E^{*} / 3$ and $m^{*}<E^{*} / 10$. In Figs. 2, $3,4,5,6,7,8$ and 9 we show the effect of these kinematic constraints on the quality of fragmentation approximation.

The results obtained for baryons with different spin, $\boldsymbol{\Xi}_{c c 0}$ and $\Xi_{c c 1}$, are very similar to each other. We sum them together under the name of $\Xi_{c c}$ (i.e., $d \sigma\left(\Xi_{c c}\right)=d \sigma\left(\Xi_{c c 0}\right)+$ $d \sigma\left(\Xi_{c c 1}\right)$ ). The behavior of interparticle correlations (such as the separation in rapidities or azimuthal angles) is also similar in all cases, for all of the considered processes (5)-(7). We only show the $\Xi_{c c}$ sample as a representative example.

With harder cuts on the fragmenting quark transverse momenta, the system becomes better collimated (narrower $\Delta \phi$ and $\Delta \eta$ distributions, see Figs. 2, 3) and so, better suits the fragmentation topology. Imposing cuts on the invariant mass makes the $\Delta \phi$ and $\Delta \eta$ distributions even narrower.

The quality of the fragmentation approximation can further be inspected by comparing the distributions on the jet invariant mass $m^{*}$ and the fragmentation variable $z$. The 'full' results for unrestricted $p_{\psi T}$ and $m^{*}$ (dotted curves in Figs. 4, $5,6,7,8$ and 9) lie well above the fragmentation predictions
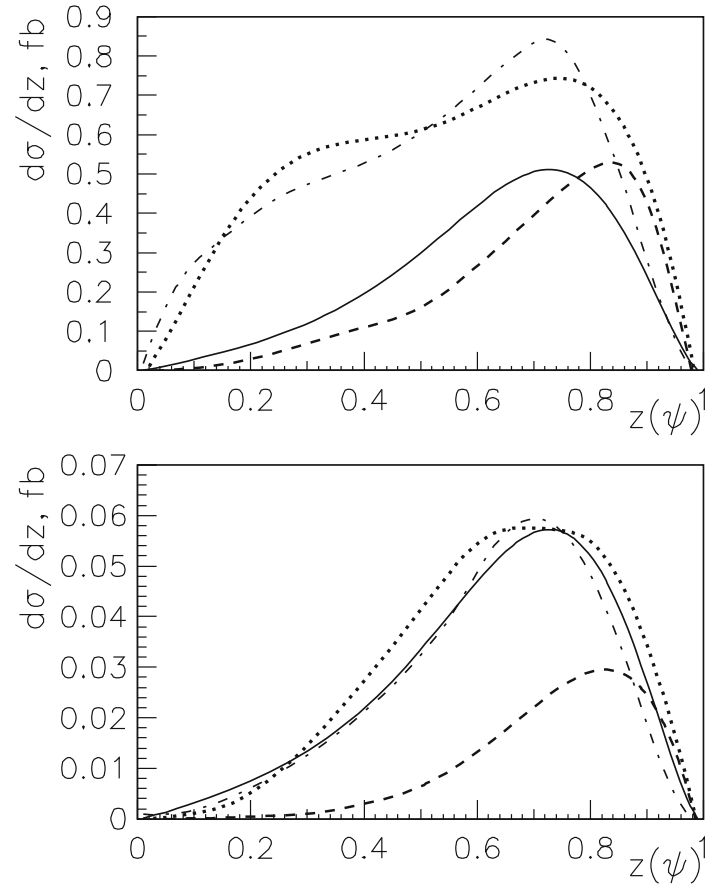

Fig. 9 The process $\gamma+\gamma \rightarrow J / \psi+c+\bar{c}$; the distributions over the fragmentation variable $z$ as seen under the different kinematic constraints. Upper panel: dotted, $p_{\psi T}>5 \mathrm{GeV}, p_{T}^{*}>20 \mathrm{GeV}$; dashed, $p_{\psi T}>20 \mathrm{GeV}, \quad p_{T}^{*}>20 \mathrm{GeV}$; dash-dotted, $m^{*}<E^{*} / 3$; solid, factorization calculation for $p_{T}^{*}>20 \mathrm{GeV}$. Lower panel: dotted, $p_{\psi T}>20 \mathrm{GeV}, p_{T}^{*}>50 \mathrm{GeV}$; dashed, $p_{\psi T}>50 \mathrm{GeV}$, $p_{T}^{*}>50 \mathrm{GeV}$; dash-dotted, $m^{*}<E^{*} / 10$; solid, factorization calculation for $p_{T}^{*}>50 \mathrm{GeV}$

(solid curves). The excess is clearly seen even in the very forward region (at large $z$ ) for $p_{T}^{*}>20 \mathrm{GeV}$ and, to a less extent, for $p_{T}^{*}>50 \mathrm{GeV}$.

Imposing restrictions on the jet invariant mass $m^{*}$ improves the line shape of $z$ distributions. A good agreement with the fragmentation predictions is obtained even under such a moderate condition as $m^{*}<E^{*} / 3$. At $m^{*}<E^{*} / 10$, the agreement can be said perfect. The invariant mass spectrum is narrower than in the true fragmentation, because the condition $m^{*} \ll E^{*}$ suppresses the large-mass tail of the spectrum.

So, we see that with tighter cuts on $p_{T}^{*}$ and $m^{*}$ we better fit the factorization conditions and better reproduce the shape of the fragmentation function. The point of difficulty is that $p_{T}^{*}$ and $m^{*}$ are not experimental observables. In our real life, in inclusive measurements, we are left with the momentum of the only reconstructed particle. Finally, in Fig. 10 we plot the calculated $p_{T}$ spectra of the different particles produced in $p p$ or $e e$ collisions at $\sqrt{s}=7 \mathrm{TeV}$. The 'full LO' and 'fragmentation' curves seem to join at around $p_{\psi T} \simeq 40$ or $50 \mathrm{GeV}$. This figure indicates that making use of the fragmentation approach below $40 \mathrm{GeV}$ is by no means well justified. But even at $p_{\psi T}>50 \mathrm{GeV}$ the apparent agreement between the spectra yet does not provide an evidence of the true factor- 

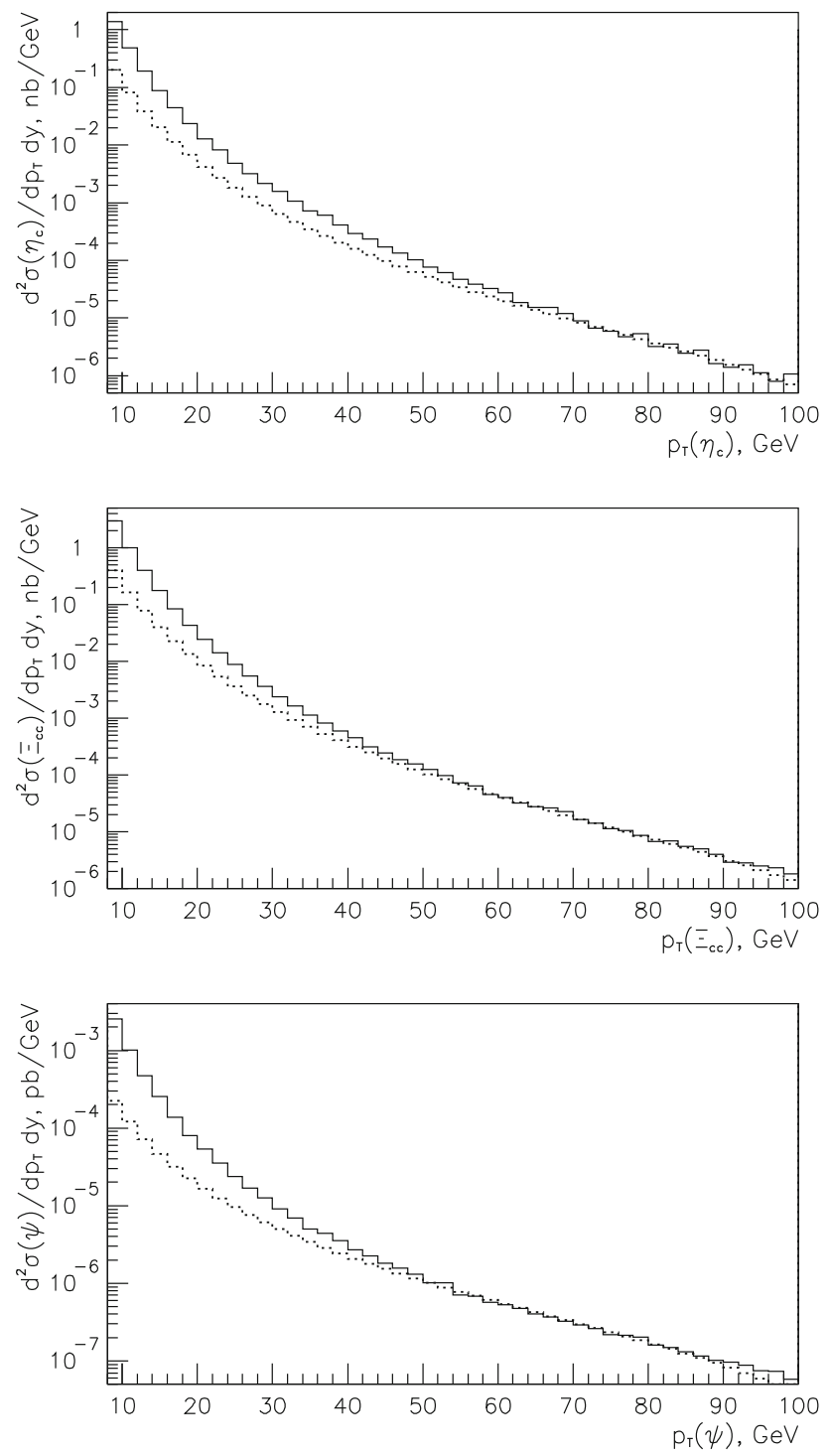

Fig. 10 Transverse momentum distributions of the different particles produced at the middle rapidity in $p p$ or $e e$ collisions at $\sqrt{s}=7 \mathrm{TeV}$. The full $\mathcal{O}\left(\alpha_{s}^{4}\right)$ calculation is shown by solid curves. The fragmentation approximation is presented by dashed curves. The plots from top to bottom correspond to the subprocesses $g+g \rightarrow \eta_{c}+c+\bar{c}, \quad g+g \rightarrow$ $\Xi_{c c}+\bar{c}+\bar{c}$, and $\gamma+\gamma \rightarrow J / \psi+c+\bar{c}$

ization (recall the disagreement between the $z$ distributions at $p_{\psi T}>50 \mathrm{GeV}$ for moderate and low $z$ in Figs. 5, 7). It is rather a consequence of the steep $p_{T}$ dependence of the production cross sections that makes the low- $z$ behavior of $D(z)$ not visible under the contributions from lower $p_{T}$.

Going to higher order calculations for the charm fragmentation function would not help, since the origin of the problem is not in the accuracy of the fragmentation function on its own, but rather in the unavoidable presence of large non-fragmentation contributions. Inclusion of the color octet production scheme cannot help either, as it would not solve the problem in the color singlet channel and, most probably, will suffer from the same troubles, in view of even much larger number of non-fragmentation diagrams.

\section{Conclusions}

We have compared the predictions on the production of $\eta_{c}+c+\bar{c}, \quad \Xi_{c c}+\bar{c}+\bar{c}$, and $J / \psi+c+\bar{c}$ systems in $p p$ collisions obtained, on one hand, with the full LO set of diagrams and, on the other hand, with the sole fragmentation mechanism. The non-fragmentation contributions are found to be rather large, extending up to as high transverse momenta as about $\sim 40 \mathrm{GeV}$. These contributions significantly change the slope of the transverse momentum spectra in the intermediate region (between 10 and $40 \mathrm{GeV}$ ). The accuracy of the fragmentation approximation can neither be improved with more precise calculations of the charm fragmentation function, nor by including the color octet production channels. The presence of essentially non-fragmentation contributions makes the fragmentation approximation for the considered processes below $40 \mathrm{GeV}$ not trustworthy.

Acknowledgements This work was supported in part by Fondecyt grants 1170319 (Chile), by Proyecto Basal FB 0821 (Chile), and by CONICYT grant PIA ACT1406 (Chile).

Data Availability Statement This manuscript has no associated data or the data will not be deposited. [Authors' comment: This is a theoretical study and no experimental data has been listed.]

Open Access This article is licensed under a Creative Commons Attribution 4.0 International License, which permits use, sharing, adaptation, distribution and reproduction in any medium or format, as long as you give appropriate credit to the original author(s) and the source, provide a link to the Creative Commons licence, and indicate if changes were made. The images or other third party material in this article are included in the article's Creative Commons licence, unless indicated otherwise in a credit line to the material. If material is not included in the article's Creative Commons licence and your intended use is not permitted by statutory regulation or exceeds the permitted use, you will need to obtain permission directly from the copyright holder. To view a copy of this licence, visit http://creativecomm ons.org/licenses/by/4.0/.

Funded by $\mathrm{SCOAP}^{3}$.

\section{References}

1. J.C. Collins, D.E. Soper, Nucl. Phys. B 194, 445 (1982)

2. G.T. Bodwin, K.-T. Chao, H.S. Chung, U.R. Kim, J. Lee, Y.-Q. Ma, Phys. Rev. D 93, 034041 (2016)

3. Y.-Q. Ma, J.-W. Qiu, H. Zhang, Phys. Rev. D 89, 094029 (2014)

4. G.T. Bodwin, H.S. Chung, U.R. Kim, J. Lee, Phys. Rev. D 91, 074013 (2015)

5. E. Braaten, K. Cheung, T.C. Yuan, Phys. Rev. D 48, 4230 (1993)

6. Z.B. Kang, J.W. Qiu, G. Sterman, Phys. Rev. Lett. 108, 102002 (2012)

7. P. Cho, A.K. Leibovich, Phys. Rev. D 54, 6690 (1996)

8. P. Cho, A.K. Leibovich, Phys. Rev. D 53, 150 (1996) 
9. P. Artoisenet, Quarkonium production phenomenology. Doctoral Thesis, Université Catholique de Louvain (2009). http://hdl.handle. net/2078.1/24548. Accessed 27 Apr 2021

10. S.P. Baranov, B.Z. Kopeliovich, Eur. Phys. J. C 79, 241 (2019)

11. X.-C. Zheng, C.-H. Chang, X.-G. Wu, Phys. Rev. D 100, 014005 (2019)

12. J.P. Ma, Z.G. Si, Phys. Lett. B 568, 135 (2003)

13. E. Bycling, K. Kajantie, Particle Kinematics (Wiley, New York, 1973)

14. S.P. Baranov, Phys. Rev. D 73, 074021 (2006)
15. S.P. Baranov, Phys. Rev. D 54, 3228 (1996)

16. J.A.M. Vermaseren, Symbolic Manipulations with FORM (Computer Algebra Nederland, Amsterdam, 1991). ISBN:90-74116-019

17. A.D. Martin, W.J. Stirling, R.S. Thorne, G. Watt, Eur. Phys. J. C 63, 189 (2009)

18. C.F. Weizsäcker, Z. Phys. C 88, 612 (1934)

19. E.J. Williams, Phys. Rev. 45, 729 (1934)

20. G.P. Lepage, J. Comput. Phys. 27, 192 (1978) 